\title{
CONTRIBUIÇÃO DA MODELAGEM BIM PARA FACILITAR O PROCESSO DE ACV DE EDIFICAÇÕES COMPLETAS
}

\author{
CONTRIBUTION OF BIM-AIDED DESIGN TO FACILITATE \\ THE LIFE CYCLE ASSESSMENT OF WHOLE BUILDINGS
}

\author{
Vanessa Gomes', Natalia Nakamura Barros ${ }^{1}$
}

RESUMO: A combinação de estratégias de projeto sustentável e BIM pode mudar as práticas tradicionais e produzir de forma eficiente um projeto de alto desempenho, mas ainda esbarra em dificuldades de interoperabilidade de software. A extração automática é o mais citado dentre os apoios potenciais que modelos BIM oferecem à realização de análises ambientais. O exame da literatura não confirma a validação desse procedimento por especialistas de avaliação do ciclo de vida (ACV), mas registra estudos incipientes para aumentar a integração de BIM e ACV. Este artigo explora contribuições da modelagem BIM para facilitar o processo de ACV de edificações completas. Procura-se verificar as hipóteses de que (1) a extração automática de quantitativo de materiais a partir de modelo com nível de desenvolvimento (LOD) 300 contribui positivamente para a realização de ACV (BIM+LCA) e (2) a inserção de parâmetros ambientais diretamente no modelo e na execução de cálculos dentro do próprio programa BIM (BIM4LCA) pode aumentar significativamente essa contribuição. Utilizou-se o software BIM Revit Architecture 2016 para modelagem dos elementos arquitetônicos, estruturais, hidrossanitários e de condicionamento artificial. Analisamos as variações entre a quantificação de referência - realizada manualmente, a partir de documentos de projeto - e aquela obtida por extração automática, assim como a adequabilidade do LOD utilizado para fins de ACV. A variação discreta (até 12\%) entre as quantificações confirma a validade da extração automática para realização de ACV e da modelagem em LOD 300 para o nível de acurácia praticado atualmente. Na segunda parte do estudo, demonstramos que modelos BIM podem ser preparados para facilitar ACV (BIM4LCA) de forma operacionalmente simples, mas de grande efetividade. Em novos desenvolvimentos de projetos, esse modo pode fornecer feedback rapidamente - ainda que unidirecional - e fundamentar uma tomada de decisão mais robusta para elevar desempenho ambiental de edificações, enquanto o emprego de tecnologias de "extração, transformação e carregamento" (ETL) ou a interoperabilidade completa não se tornarem prática comum.

PALAVRAS-CHAVE: BIM; ACV; Integração; Extração Automática; Mapa de Processo.

ABSTRACT: Combining sustainable design strategies and BIM can change traditional practices and efficiently lead to high-performance designs, however, this is still impaired by deficient software interoperability. Automatic extraction is one of the most cited ways that BIM models can support environmental analyses. Though the reviewed literature does not confirm validation of this procedure by life cycle assessment (LCA) experts, it presents incipient procedures to systematically increase the use of BIM for LCA. This article explores the contributions offered by BIM to facilitate and improve the LCA of whole buildings. Our study sought to verify the hypotheses that: (1) the quantitative automatic extraction of the bills of materials from BIM models with a level of development (LOD) 300 positively contributes to LCA processes (BIM+LCA); and (2) embed ding environmental parameters and calculations within the model to extract calculated impacts (BIM4LCA) can significantly improve such contribution. We used the software Revit Architecture 2016 to model the architectural, structural, sanitary and HVAC elements. We analyzed the variations between the baseline (manually performed, based on design documents) and BIM-supported automatically extracted bill of materials, as well as the suitability of the used LOD for LCA. We also showed that BIM models can be prepared to facilitate LCA (BIM4LCA) through a low complexity,

\section{How to cite this article:}

GOMES, V.; BARROS, N. N. Contribuição da modelagem BIM para facilitar o processo de ACV de edificações completas. Gestão e Tecnologia de Projetos, São Carlos, v. 13, n. 2, p. 19-34, 2018 http://dx.doi.org/10.11606/gtp.v13i2.142139

Fonte de financiamento: Declaram não haver Conflito de interesse: Declaram não haver Submetido em: 31/12/2017 Aceito em: 06/03/2018 
high effectiveness operational measure. This procedure can provide reasonably quick feedback to support decision-making and enhance the environmental performance of new building designs, until 'extraction, transformation and loading' (ETL) technologies or full interoperability become mainstream practice.

KEYWORDS: BIM; LCA; Integration; Automatic Extraction; Process Mapping

\section{INTRODUÇÃO}

Os impactos relacionados à produção e utilização de edificações são significativos e, mundialmente, correspondem a mais de $40 \%$ do consumo de energia e cerca de $30 \%$ das emissões de gases de efeito estufa (IPCC, 2014; UNEP, 2009a; UNEP, 2009b).

As estratégias delineadas para sustentabilidade de edificações permeiam diferentes campos de atuação e envolvem múltiplos agentes. Da perspectiva dos projetistas, uma das barreiras mais importantes a transpor consiste no desconhecimento do desempenho ambiental de soluções à medida em que são incorporadas a um projeto, principalmente em seus estágios iniciais de desenvolvimento. A limitação de informação disponível no ambiente de trabalho e formato familiar aos projetistas impedem as iterações necessárias para testar e modificar proposições até se chegar a um desempenho ambiental otimizado e satisfatório.

A modelagem de informação da construção (BIM) pode ser definida como um processo de geração, gestão, troca e compartilhamento de informações multidisciplinares da construção de forma interoperável e reutilizável (VANLANDE; NICOLLE; CRUZ, 2008), que oferece benefícios em todas as fases do ciclo de vida de um empreendimento (BECERIK-GERBER; KENSEK, 2010; BYNUM; ISSA; OLBINA, 2013; MEADATI; IRIZARRY; AKHNOUKH, 2011).

A combinação de estratégias de projeto sustentável e de modelagem de informação da construção torna-se especialmente útil para avaliar aspectos de sustentabilidade e apoiar a tomada de decisão correspondente durante o desenvolvimento de projeto (SCHADE et al., 2013; WONG; FAN, 2013), particularmente no que toca a seleção de materiais, redução do consumo material e aumento no conteúdo reciclado (KRYGIEL; NIES, 2008), com potencial de economia significativa de tempo e recursos (AZHAR, 2010) e transformar radicalmente as práticas tradicionais (AZHAR et al., 2011).

A avaliação de ciclo de vida (ACV ou LCA, da expressão "life cycle assessment") é fundamental para alcançar as metas estabelecidas globalmente para diminuir os impactos dos edifícios. A utilização de tal procedimento na prática projetual é uma das principais peças que faltam para permitir a responsabilização ambiental rápida de decisões de projeto e para orientar a tomada de decisões na busca de soluções avançadas. BIM pode ajudar e deve ser explorado nesse sentido.

$\mathrm{Na}$ ACV tradicional, uma vez que o objetivo é definido, todos os quantitativos são extraídos manualmente e só então a avaliação é processada em software especializado. Se há mudanças na solução, a listagem de material deve ser reprocessada a cada vez. A interoperabilidade completa entre BIM e ACV permitiria exportação direta de informações do modelo para realizar cálculo ambientais numa plataforma ACV, que, por sua vez, devolveria automaticamente os resultados para avaliação no ambiente BIM.

Enquanto esse cenário ideal não se concretiza através de programas e/ ou plug-ins, soluções intermediárias podem facilitar o processo de avaliação ambiental. A mais simples delas consiste na extração automática de informações a partir do modelo, como quantitativos de matérias, por exemplo. 0 principal impacto do procedimento de extração automática de quantitativos de materiais ocorre na etapa de análise de inventário da ACV, especificamente durante a coleta de dados da edificação. Havendo alterações no modelo do projeto, os quantitativos de materiais são atualizados automaticamente, sem repetir a demorada tarefa de coletar dados manualmente a partir dos projetos 2D. 
No entanto, apesar de estudos confirmarem essa possibilidade (ALWAN; JONES, 2014; DIAZ; ANTON, 2014; IDDON; FIRTH, 2013; PENG, 2014; SHADRAM et al.,2015), ao realizarem a revisão sistemática de literatura, Barros e Silva (2016) não encontraram registros de validação desse procedimento por analistas de ACV, e a ênfase dos trabalhos mantém-se na perspectiva de desenvolvimentos em BIM.

Para auxiliar no preenchimento dessa lacuna de conhecimento, este artigo explora contribuições da modelagem BIM para facilitar o processo de ACV de edificações completas. Procura-se verificar as hipóteses de que (1) a extração automática de quantitativo de materiais a partir de modelo com nível de desenvolvimento (LOD) 300 contribui positivamente para a realização de ACV (BIM+LCA) e (2) a inserção de parâmetros ambientais diretamente no modelo e execução de cálculos dentro do próprio programa BIM (BIM4LCA) é uma medida operacionalmente simples, mas de grande impacto positivo, capaz de fornecer feedback rápido e fundamentar uma tomada de decisão mais robusta.

\section{AVALIAÇÃO DO CICLO DE VIDA DE EDIFICAÇÕES - ACV (LIFE CYCLE ASSESSMENT - LCA)}

A técnica de ACV modela o ciclo de vida de um produto por meio de seu sistema de produto, que consiste nas fases pelas quais o sistema passará do berço ao túmulo ou "fim de vida" (SAADE, 2013). A NBR ISO 14044 divide a realização de ACV em quatro fases: definição do objetivo e escopo, análise de inventário, avaliação de impacto e interpretação (ASSOCIAÇÃO BRASILEIRA DE NORMAS TÉCNICAS, 2009a).

O objetivo e o escopo devem declarar a aplicação pretendida, as razões para conduzir o estudo e o público-alvo, além de descrever claramente o procedimento de condução da ACV (ASSOCIAÇÃO BRASILEIRA DE NORMAS TÉCNICAS, 2009b).

A análise de inventário do ciclo de vida (ICV) envolve a coleta de dados e procedimentos de cálculo para quantificar as entradas e saídas pertinentes de um sistema de produto. Dados qualitativos e quantitativos para inclusão no inventário são coletados para cada unidade de processo que esteja incluída dentro das fronteiras de sistema estabelecidas. Os procedimentos usados para a coleta de dados podem variar dependendo do escopo, da unidade de processo ou da aplicação pretendida para o estudo. Esses dados constituem a entrada para a avaliação do impacto do ciclo de vida (ASSOCIAÇÃO BRASILEIRA DE NORMAS TÉCNICAS, 2009b). O processo de condução de uma análise do inventário é iterativo. Na medida em que os dados são coletados e se conhece melhor o sistema, podem ser identificados novos requisitos ou limitações que requeiram revisões do estudo e mudança nos procedimentos de coleta (ASSOCIAÇÃO BRASILEIRA DE NORMAS TÉCNICAS, 2009b).

A fase de avaliação do impacto da ACV trata da relevância de impactos ambientais potenciais, a partir dos resultados da análise de inventário. $\mathrm{O}$ objetivo dessa etapa é explicitar os impactos decorrentes das cargas ambientais calculadas na análise de inventário, transformando os resultados da etapa anterior em resultados ambientalmente relevantes, relacionando-os a impactos específicos, como acidificação e depleção da camada de ozônio (BAUMANN; TILLMAN, 2004). Nível de detalhamento, escolha dos impactos avaliados e metodologias utilizadas dependem do objetivo e do escopo do estudo (ASSOCIAÇÃO BRASILEIRA DE NORMAS TÉCNICAS, 2009b).

\section{MODELAGEM DE INFORMAÇÃO DA CONSTRUÇÃO (BIM)}

A modelagem de edifícios baseada em sólidos 3D foi desenvolvida no final da década de 1970 e início da de 1980. A atual geração de ferramentas BIM para projetos de arquitetura desenvolveu-se a partir das capacidades da modelagem paramétrica baseada em objetos e foi desenvolvida inicialmente 
para sistemas mecânicos. Objetos paramétricos podem ser caracterizados como objetos que possuem dados e regras associados que compõem geometrias integradas, sem inconsistências, e que permitem a modificação automática. Eles possibilitam diferentes níveis de agregação, podendo vincular-se a ou receber, divulgar ou exportar conjuntos de atributos (EASTMAN et al., 2008).

Os modelos BIM podem ser caracterizados pelo nível de desenvolvimento (LOD) em que eles se encontram. O LOD descreve o nível mínimo de informação dimensional, espacial, quantitativa, qualitativa, entre outras, incluído em um elemento de modelo, e apoia os usos autorizados associados a cada LOD. É uma medida que reflete a maturidade da informação e sua riqueza e está presente ao longo de todas as fases do projeto (AMERICAN INSTITUTE OF ARCHITECTS, 2013).

O American Institute of Architects (AIA) desenvolveu o seu primeiro conjunto de definições de LOD em 2008, no documento AIA Document E202 - Building information modeling protocol exhibit. Devido à rápida evolução do uso de BIM, em 2013 o AIA revisou a E202 e publicou os documentos AIA Document E203: Building information modeling and digital data exhibit; AIA Document G201: Project digital data protocol form e AIA Document G202: Project building information modeling protocol form, que são acompanhados por um guia (BEDRICK, 2013). Cada LOD especifica um tipo de modelo e mostra em qual nível se encontra (AMERICAN INSTITUTE OF ARCHITECTS, 2013), facilitando a troca de informações entre os agentes envolvidos no processo de projeto da edificação:

- O LOD 100 é equivalente ao projeto conceitual. O modelo consiste em um indicativo de área, altura, volume, localização e orientação que podem ser modelados em três dimensões ou representados por outros dados. Ele pode ser utilizado para análise conceitual, para estimativas de custos primárias e para planejamento da obra e duração total em termos gerais.

- O LOD 200 é equivalente ao desenho esquemático, no qual os elementos são modelados como sistemas generalizados ou conjuntos com quantidades aproximadas, tamanho, forma, localização e orientação. Esse modelo pode ser utilizado para análises aproximadas de desempenho de sistemas a partir da aplicação de critérios gerais, para estimativas de custos, estabelecimento de quantitativos e durações para a instalação, bem como os prazos de entrega dos equipamentos.

- No LOD 300 os elementos são modelados como conjuntos específicos precisos em termos de quantidade, tamanho, forma, localização e orientação. É adequado para a geração de documentos de construção. Além disso, esse modelo pode ser utilizado para análise detalhada de sistemas de condicionamento artificial, determinação da carga e simulação estrutural, estimativa de custos precisa, mostrar a aparência em escala de tempo de elementos detalhados e sistemas e para a detecção de conflitos.

- No LOD 400 os elementos são modelados com medidas precisas em termos de tamanho, forma, localização, quantidade e orientação com detalhamento da fabricação completa, montagem e informações. Nesse, os elementos do modelo são representações virtuais do elemento proposto e são adequados para construção. Esse modelo pode ser utilizado para análise de desempenho aprovado, estimativas de custos reais e mostrar os meios e métodos de construção da edificação.

- O LOD 500 pode ser visto como um modelo as built, no qual os elementos são modelados como conjuntos reais e precisos em termos de tamanho, 
forma, localização, quantidade e orientação. Pode ser utilizado para manutenção, alteração e modificação no projeto, gestão de programas de manutenção programada e reacionária que podem ser catalogados e arquivados durante toda a vida do edifício e reformas futuras, usando o modelo como base para projetar as próximas modificações.

O propósito da utilização do modelo BIM (por exemplo, em estimativa de custos, simulação de energia e criação de desenhos de fabricação) é o principal definidor do seu LOD (LEITE et al., 2011). Mesmo para apoiar a realização de ACV, o LOD do modelo BIM pode variar, dependendo do objetivo e escopo para elas estabelecido.

\section{ESTADO ATUAL E DESAFIOS PARA INTEGRAÇÃO DE ACV E BIM}

Na prática atual de ACV, o analista inspeciona os documentos de projeto e construção manualmente para determinar os insumos materiais, estimar o consumo de energia operacional e, em seguida, transferi-los para algum software de cálculo específico (DIAZ; ANTON, 2014; RIST, 2011). Essa avaliação - quando realizada - ocorre geralmente depois que o projeto da edificação está completo, e alterações são caras e de difícil implementação (DAWOOD et al., 2009), ainda que os impactos ambientais de uma edificação sejam determinados principalmente a partir de decisões tomadas nos estágios iniciais de projeto (BASBAGILL et al., 2013; DIAZ; ANTON, 2014; KULAHCIOGLU et al., 2012). Com isso, perde-se o principal benefício conceitual de processos de projeto apoiados por BIM: compartilhamento de informações multidisciplinares em um único ambiente que permita múltiplos ciclos de quantificação, análise e intervenção até convergir para uma solução de projeto ambientalmente otimizada.

A integração de ACV-BIM permitiria estimar rapidamente impactos ambientais relevantes (IDDON; FIRTH, 2013) e retornar essas informações ao projetista para embasar e refinar suas especificações de materiais e componentes iterativamente no desenvolvimento de projetos. No entanto, essa ainda não é uma prática possível, principalmente pela falta de informações ambientais sobre os materiais e componentes armazenados no banco de dados; e de interoperabilidade entre as ferramentas de projeto e de análises ambientais de edifícios (BECERIK-GERBER; KENSEK, 2010; JRADE; JALAEI, 2013).

Como a etapa de análise de inventário concentra a maior parte do tempo e esforço empreendidos na condução de uma ACV, ferramentas que automatizem o levantamento de dados são particularmente benéficas. Se os objetos que compõem o modelo BIM já contiverem as informações ambientais necessárias, grande parte dos dados de materiais podem ser exportados automaticamente do modelo (YUNG; WANG, 2014). Essas informações ambientais precisam ser retiradas de bases de dados de inventários de ciclo de vida, levantados para o contexto de avaliação. Esta é uma das maiores limitações intrínsecas à técnica de ACV e, portanto, fora do escopo deste trabalho.

Os desenvolvimentos recentes em interoperabilidade têm focado na interação com software para simulação de desempenho energético operacional, e a interoperabilidade prática com as ferramentas especializadas de ACV segue negligenciada (SHADRAM et al., 2016). Isso aumenta tempo, esforço e risco de erros durante a reinserção manual de dados, seja no modelo ou em software especializado, e dificulta a otimização integrada de impactos incorporados e operacionais (SHADRAM; MUKKAVAARA, 2018).

Publicações específicas na interface ACV-BIM concentram-se na aplicação simplificada de ACV, normalmente enfocando emissões de $\mathrm{CO}_{2}$ nos estágios iniciais de projeto (SOUST-VERDAGUER; LLATAS; GARCÍA-MARTÍNEZ, 2017), e em métodos para integrar, em processos de projeto apoiados por BIM, ferramentas convencionais de ACV (estágio BIM+LCA, na Figura 1), ainda incapazes de distinguir diferentes fornecedores e cadeias de suprimentos e sem incorporar consistentemente dados de produtos específicos verificados como declarações ambientais de produtos (EPD), por exemplo (SHADRAM et 
al., 2016). Shadram et al. (2016), Barros e Silva (2016), e Shadram e Mukkavaara (2018) apresentam bons sumários das principais iniciativas.

Até que as dificuldades de interoperabilidade de software sejam sanadas, as tecnologias de "extração, transformação e carregamento" (ETL, da expressão "extraction, transformation and loading") constituem uma oportunidade importante para aumentar a integração ACV-BIM (estágio 3 na Figura 1).

Ferramentas ETL estabelecem processos repetitivos para extrair dados de fontes múltiplas e heterogêneas; transformar os dados extraídos em um formato ou estrutura consistente; e finalmente carregar os dados transformados de volta a base de dados ou aplicativo (SHADRAM; MUKKAVAARA, 2018). Recursos ETL já foram usados em outras disciplinas para superar problemas de interoperabilidade e gerenciar grandes conjuntos de dados contendo informações de múltiplas fontes, que podem ser gravadas em diferentes formatos, usando diferentes modelos de dados (BIMONTE et al., 2014, KIMBALL; CASERTA, 2004), o que sugere potencial para utilização no setor de arquitetura, engenharia, construção e operação (Aeco). No entanto, o uso de ETL no contexto de BIM ainda é pouco explorado (SHADRAM; MUKKAVAARA, 2018).

Nesse sentido, destaca-se a framework proposta por Shadram et al. (2016) para que definições de projeto fossem feitas paralelamente ao emprego de processos ETL para automatizar o cálculo de impactos incorporados em componentes, a partir de EPD de produtos específicos, e rastrear a distância entre esses fornecedores e o local da construção. A substituição de componentes na biblioteca usada no modelo BIM permitiria atualizar as informações necessárias ao cálculo dos impactos incorporados e de transporte.

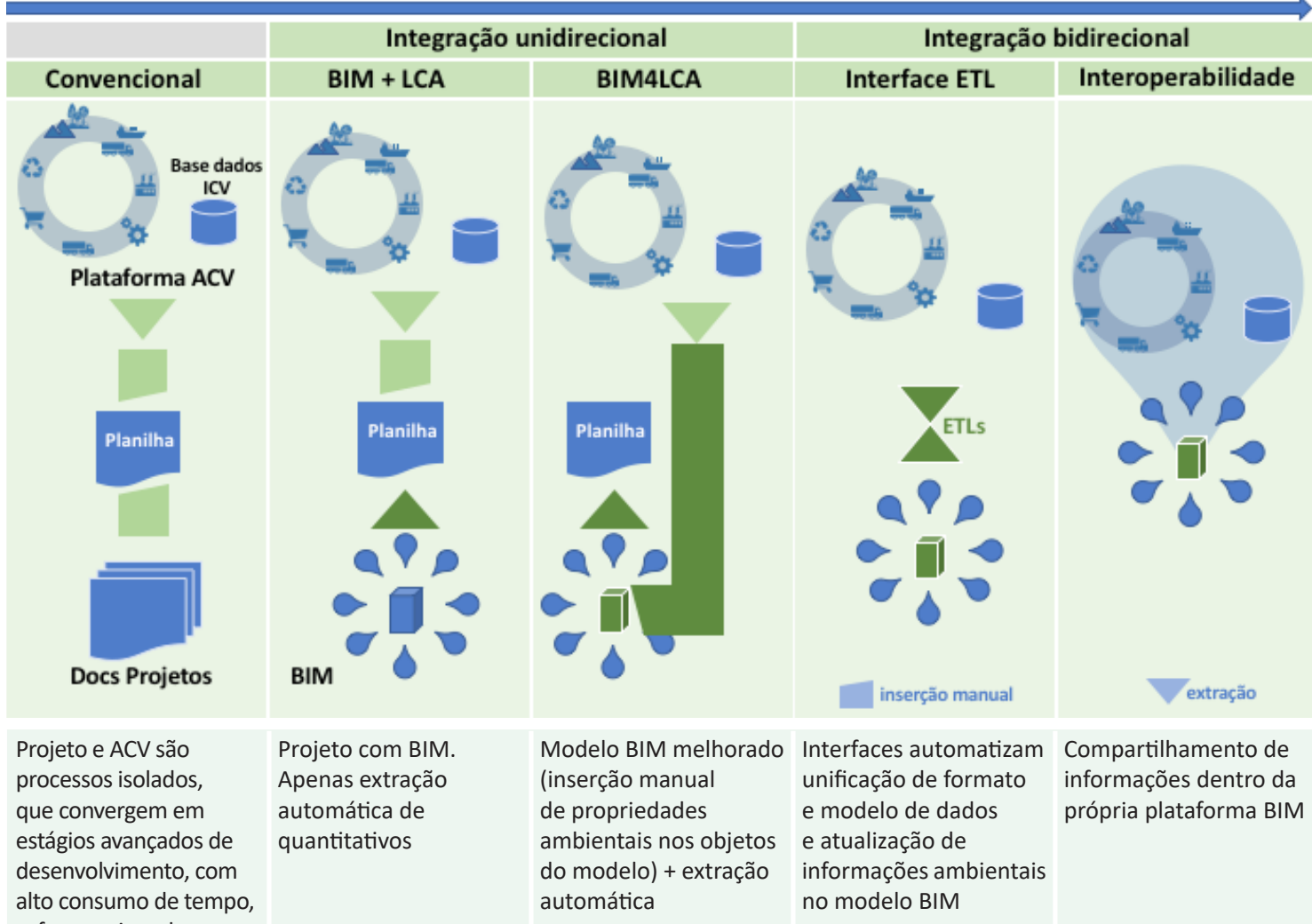

esforço e risco de erros

Figura 1: Estágios de integração entre ACV e BIM

Fonte: Elaborado pelas autoras
Utilizar EPD é um refinamento apropriado para situações com LOD tal que os elementos modelados sejam representações virtuais do elemento proposto, o que tipicamente ocorre em fases avançadas do desenvolvimento do projeto. Os processos ETL propostos por aqueles autores garantem fluidez de atualização dos componentes do modelo à medida em que sua definição 
evolui, mas não se enquadram bem a etapas anteriores do processo de projeto ou a modelos com LOD inferiores. Nessas situações, dados genéricos médios de ACV são suficientes e incomparavelmente mais abundantes que EPD, e o emprego de funções para extração automática de quantitativos diretamente do modelo BIM representa um estágio anterior de integração, que merece ser explorado.

\section{MÉTODO}

Para alcançar o objetivo estabelecido, esta pesquisa foi desenvolvida em duas partes: primeiramente, um estudo comparativo entre processos de quantificação manual a partir de documentos de projeto (referência) e de extração automática de quantitativos de modelo BIM; seguido de um estudo exploratório para verificar a possibilidade de melhorias no segundo procedimento. $\mathrm{O}$ estudo comparativo compreendeu cinco etapas:

1) Análise da documentação das disciplinas de projeto consideradas (arquitetônico, estrutural, hidrossanitário e condicionamento artificial) para o estudo de caso selecionado: um edifício institucional projetado segundo metas de elevado desempenho ambiental;

2) Definição do quantitativo de referência (baseline) a partir da revisão do levantamento feito manualmente por Silva et al. (2014) para ACV do mesmo estudo de caso;

3) Modelagem dos elementos dos projetos no LOD 300, utilizando a ferramenta BIM Revit Architecture 2016;

4) Extração automática de quantitativos a partir do modelo de informação (estágio BIM+LCA, na Figura 1);

5) Comparação dos resultados dos dois procedimentos - referência versus apoiado por BIM - para quantificação de materiais.

Já no estudo exploratório (etapa 6), avançamos o emprego de recursos de modelagem de informação para inserir parâmetros ambientais diretamente no modelo BIM (estágio BIM4LCA, na Figura 1). Procedeu-se a análise do mapeamento do processo resultante em notação BPMN (business process model and notation), que fornece um padrão gráfico compreensível por todos os agentes interessados (OBJECT MANAGEMENT GROUP, 2011).

\section{Descrição do estudo de caso}

O estudo de caso selecionado foi o projeto do Living Lab (Figura 2) projetado para a Faculdade de Engenharia Civil, Arquitetura e Urbanismo da Universidade Estadual de Campinas (Unicamp). O processo de projeto foi pautado por metas de altíssimo desempenho, para produzir uma edificação com caráter de demonstração e "vitrine tecnológica", oferecendo suporte experimental para coberturas verdes, condicionamento inovador e geração on-site, “mockups" de sistemas prediais para demonstração em tempo real, sistema de captação e recuperação de fontes alternativas de água, em uma estrutura visitável para estudantes, pesquisadores e profissionais. 

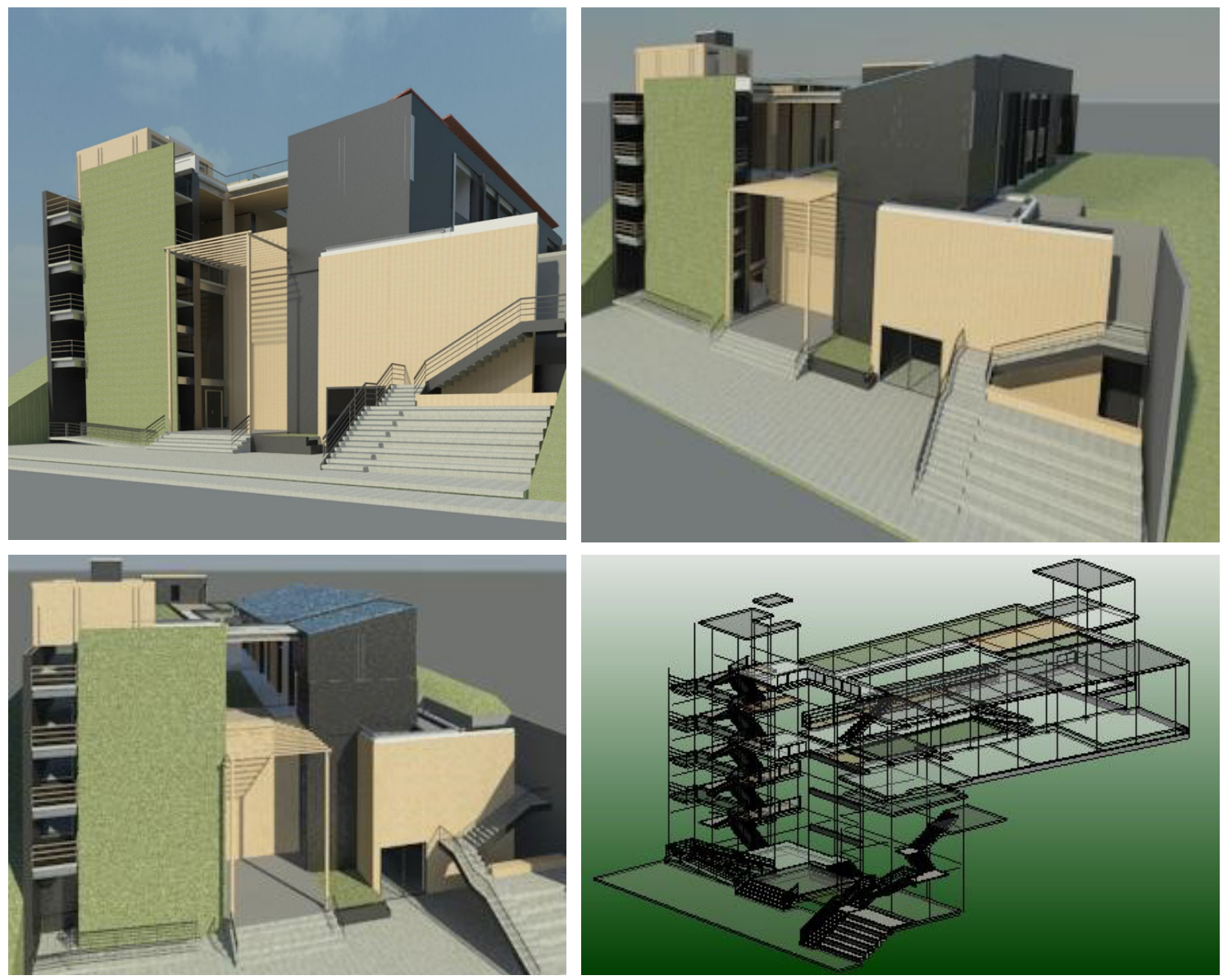

Figura 2: Imagens do modelo BIM do estudo de caso

Fonte: Elaborado pelas autoras
O Living Lab possui a fachada frontal orientada para oeste, que recebeu uma parede verde. $\mathrm{O}$ edifício é composto por três pavimentos principais, inferior, térreo e cobertura, além de três mezaninos destinados à circulação e acesso aos pavimentos. $O$ pavimento inferior compreende os ambientes de acesso à edificação e circulação horizontal e vertical (escadas, rampas e elevadores) e uma microestação de tratamento de esgoto. O pavimento térreo abriga os ambientes de ocupação principal, destinados a estudo, aulas, reuniões e pesquisa, e os espaços de convivência. A cobertura é um pavimento técnico acessível a estudantes, pesquisadores e visitantes, que compreende as instalações experimentais de sistemas de painéis fotovoltaicos e telhado verde. Todas as instalações são instrumentadas para fornecer feedback de desempenho em tempo real no hall de entrada.

\section{Premissas para quantificação de referência (baseline) e modelagem BIM}

As premissas para quantificação de referência e modelagem BIM do Living Lab seguiram as normas NBR 14040 (ASSOCIAÇÃO BRASILEIRA DE NORMAS TÉCNICAS, 2009a), NBR 14044 (ASSOCIAÇÃO BRASILEIRA DE NORMAS TÉCNICAS, 2009b) e EN 15978:2011 (BRITISH STANDARDS INSTITUTION, 2011). A fronteira do sistema para a modelagem do ciclo de vida neste estudo abrange os módulos A1 a C2, mostrados em cinza na Figura 3. O transporte para o tratamento no fim da vida foi considerado, mas o processamento de resíduos e disposição final não foram incluídos no escopo de análise (SILVA et al., 2014).

O quantitativo de referência (baseline) foi definido a partir da revisão do levantamento feito manualmente por Silva et al. (2014) para ACV do mesmo estudo de caso. A análise de inventário consistiu na coleta de dados específicos 
referentes ao modelo de estudo, levando em consideração as fronteiras do sistema estabelecidas na definição do objetivo e o escopo da análise. A coleta foi realizada a partir da documentação de projetos arquitetônico, estrutural, elétrico, hidráulico e de condicionamento artificial, que caracterizava um produto entre projeto básico e executivo. O nível de desenvolvimento do projeto variava entre disciplinas, o que se refletiu na parcela de elementos em cada subsistema que puderam ser quantificados manualmente e posteriormente modelados em BIM (Figura 3).

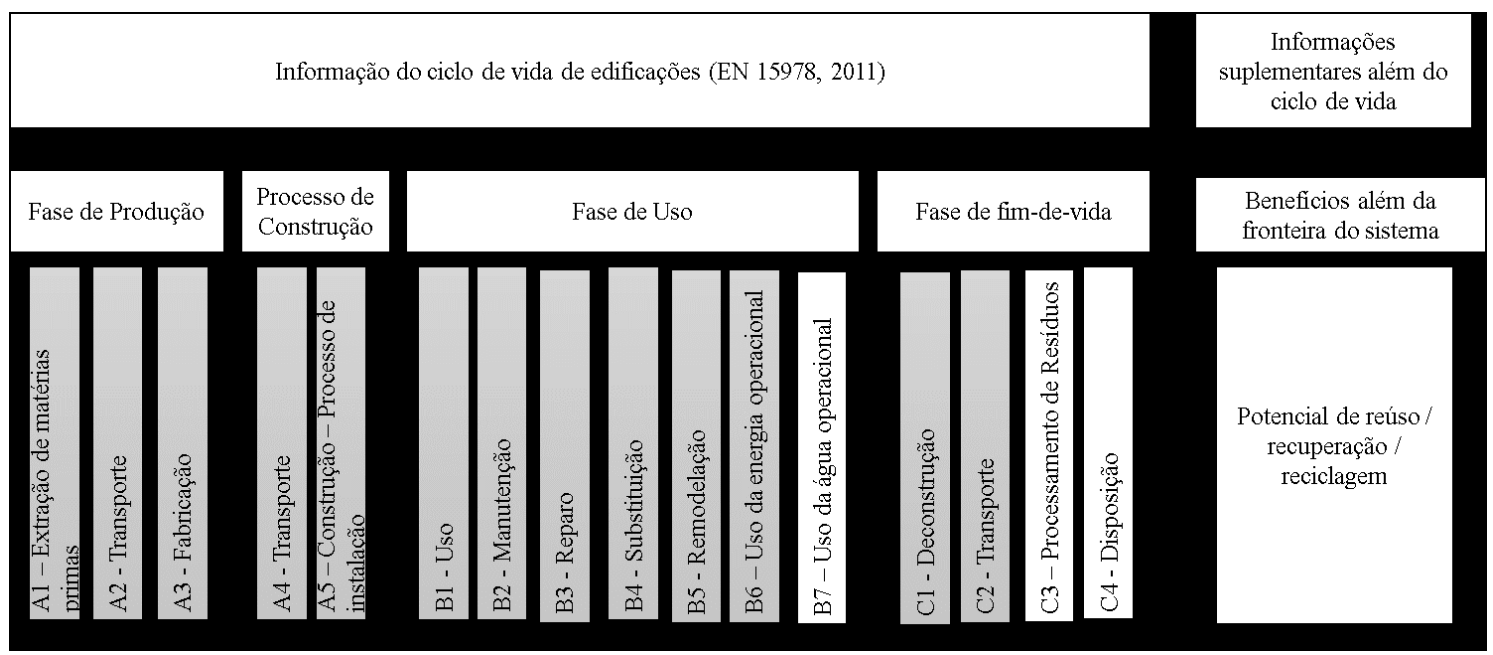

Foram computados apenas os elementos constituintes da obra bruta da edificação: paredes (sem revestimentos), pisos, sistemas estruturais, telhados, portas, janelas, sistema de tubulação hidráulica e de ar-condicionado. Equipamentos mecânicos e hidráulicos, escadas, mobiliário e acabamentos de piso não foram incluídos. Foram excluídos itens específicos do projeto analisado e indisponíveis nas bases de dados de inventário de ciclo de vida, tais como revestimento nas paredes e pisos dos sanitários; assim como aqueles excluídos com base na regra de exclusão (cutoff) aplicada, isto é: impacto $\leq 5 \%$ do impacto total e massa $\leq 1 \%$ da massa total estimados para a edificação, como no caso da madeira de reúso.

A modelagem da edificação em software BIM replicou estritamente o conteúdo, a abordagem de cálculo e o sistema de produto adotados por Silva et al. (2014), para validar a comparação entre os dois procedimentos. Utilizou-se o programa Autodesk Revit Architecture 2016 como ferramenta de apoio, por ser um software BIM dos mais utilizados por profissionais da arquitetura e construção (MCGRAW-HILL CONSTRUCTION, 2008). Os sistemas arquitetônico, estrutural, hidráulico e de ar-condicionado foram modelados segundo a organização hierárquica indicada no Quadro 1, em LOD 300.

O software Revit permite a modelagem detalhada dos elementos que compõem o modelo. $\mathrm{O}$ fator determinante do nível de detalhamento da modelagem foram, na verdade, as informações disponíveis na documentação de projeto, majoritariamente desenvolvidas ao nível de projeto básico, ou seja: com todas as suas interfaces resolvidas, de forma a permitir uma avaliação preliminar, mas sem apresentar informações técnicas claras e objetivas sobre todos os elementos, sistemas e componentes do empreendimento.

Na modelagem do sistema estrutural, isso impossibilitou a consideração de detalhamentos como parafusos e furos nas interseções entre perfis metálicos ou a modelagem das fundações do edifício. Da mesma forma, dutos de arcondicionado e tubulações hidrossanitárias foram modelados com as dimensões especificadas no projeto, porém sem considerar conexões hidrossanitárias e elementos de ligação entre dutos e equipamentos de condicionamento artificial. Finalmente, a descrição insuficiente das instalações elétricas prediais inviabilizou a modelagem do sistema: apesar de disponibilizados memoriais e tabelas de

Figura 3: Módulos de informação do ciclo de vida de edificações, segundo a EN 15978:2011

Fonte: Silva et al., 2014 
quantitativos, não constavam desenhos que indicassem pontos de iluminação, tomadas, interruptores e fiação elétrica que geraram os quantitativos.

Consideradas essas limitações, os subsistemas foram modelados em LOD 300 , restando assegurada a precisão quanto à forma, dimensão e orientação dos objetos BIM. A Figura 3 ilustra a parcela dos elementos modelados no Revit e considerados na ACV, distribuídos nos diferentes subsistemas da edificação.

Quadro 1: Organização e descrição dos elementos modelados

\begin{tabular}{|c|c|c|c|c|c|}
\hline Categoria & Família & Tipo & Estrutura & Material BIM & LOD \\
\hline \multirow{9}{*}{ Arquitetura } & \multirow{6}{*}{ Paredes } & \multirow{2}{*}{ Painel Metálico } & \multirow{2}{*}{$\begin{array}{l}0,01 \text { de aço + 0,3 de } \\
\text { poliuretano + 0,01 de aço. }\end{array}$} & $A c ̧ O-A C V$ & 300 \\
\hline & & & & Poliuretano & 300 \\
\hline & & Painel de madeira & 0,06 de madeira & Madeira - ACV & 300 \\
\hline & & Vidro & 0,1 de vidro & Vidro - ACV & 300 \\
\hline & & Placa de gesso & 0,5 de gesso & Gesso - ACV & 300 \\
\hline & & Madeira compensada & 0,6 de madeira compensada & Madeira compensada - ACV & 300 \\
\hline & Portas & \multicolumn{3}{|l|}{ De acordo com projeto } & 300 \\
\hline & Janelas & \multicolumn{3}{|l|}{ De acordo com projeto } & 300 \\
\hline & Forro & Forro de gesso & 0,05 de gesso & Gesso_forro & 300 \\
\hline \multirow{6}{*}{ Estrutura } & \multirow{2}{*}{ Pisos } & \multirow{2}{*}{ Piso estrutural } & \multirow{2}{*}{$\begin{array}{l}0,15 \text { de concreto + vergalhão } \\
\text { estrutural }\end{array}$} & Concreto CPIII-32 fck 30 & \multirow{2}{*}{300} \\
\hline & & & & Aço_vergalhão & \\
\hline & \multirow{2}{*}{ Pilares } & \multirow{2}{*}{ Pilares } & Aço & Aço_estrutura & \multirow{3}{*}{300} \\
\hline & & & Concreto & Concreto CPIII-32 fck 60 & \\
\hline & \multirow{2}{*}{ Colunas } & \multirow{2}{*}{ Colunas } & Aço & Aço_estrutura & \\
\hline & & & Concreto & Concreto CPIII-32 fck 60 & \\
\hline Hidrossanitário & Tubulação & Água potável e de reúso & De acordo com projeto & PVC/PPR & 300 \\
\hline \multirow{7}{*}{ AVAC } & \multirow{2}{*}{ Dutos } & Dutos & Aço galvanizado & Aço_galvanizado & \multirow{2}{*}{300} \\
\hline & & Isolamento & Lã de vidro & Lã de vidro & \\
\hline & \multirow{3}{*}{ Elétrica } & Eletroduto & Aço galvanizado & Aço_galvanizado (eletroduto) & 300 \\
\hline & & \multirow[b]{2}{*}{ Fios elétricos } & Cobre & Cobre (fio elétrico) & \multirow[b]{2}{*}{300} \\
\hline & & & $\begin{array}{l}\text { Isolamento de PVC + camada } \\
\text { de proteção }\end{array}$ & Isolante (fio elétrico) & \\
\hline & \multirow{2}{*}{ Hidráulica } & \multirow{2}{*}{ Tubulação de água } & Cobre & Cobre (água) & \multirow{2}{*}{300} \\
\hline & & & Aço galvanizado & Aço_galvanizado (água) & \\
\hline \multirow{2}{*}{ Telhado } & \multirow{2}{*}{ Telhado } & Telhado verde & Grama & Grama_telhado & \multirow{2}{*}{300} \\
\hline & & Telhado & Impermeabilizante & Telhado_membrana & \\
\hline
\end{tabular}

Fonte: Elaborado pelas autoras

Figura 4: Parcela dos elementos modelados no Revit e considerados na ACV

Fonte: Barros; Silva, 2016

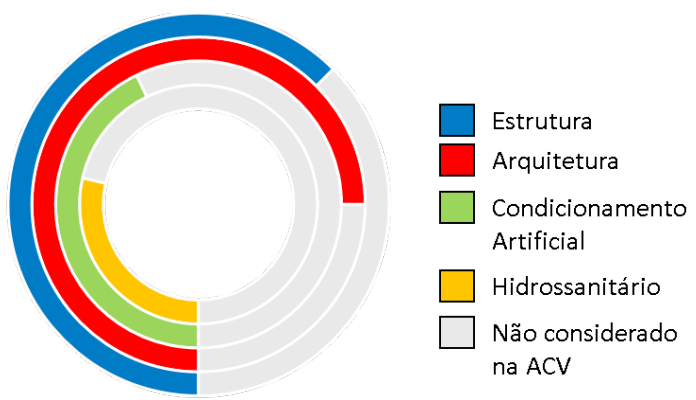

RESULTADOS E DISCUSSÃO

O quantitativo obtido pelo método com extração automática de quantitativo de materiais $(\mathrm{BIM}+\mathrm{LCA})$ apresentou valor médio superior ao calculado pelo 
procedimento de referência para a maior parte dos subsistemas analisados (Quadro 2). Isso sugere que elementos simplificados ou desconsiderados no procedimento de referência puderam ser capturados na modelagem.

Quadro 2: Comparativo entre os procedimentos de quantificação de referência e a extraída automaticamente do modelo BIM

\begin{tabular}{|c|c|c|c|c|}
\hline Subsistema & Materiais ACV & Nome BIM & Unidade & $\begin{array}{l}\text { Variação entre } \\
\text { BIM/referência* }\end{array}$ \\
\hline \multirow{4}{*}{ Estrutural } & Estrutura de aço & Aço_estrutura & $\mathrm{kg}$ & $+3 \%$ \\
\hline & Vergalhão de aço & Aço_vergalhão & $\mathrm{kg}$ & $+5 \%$ \\
\hline & Concreto CPIII-32 fck 30 & Concreto CPIII-32 fck 30 & $m^{3}$ & $+1 \%$ \\
\hline & Concreto CPIII-32 fck 60 & Concreto CPIII-32 fck 60 & $m^{3}$ & $-12 \%$ \\
\hline \multirow{3}{*}{ Fachadas } & Painel metálico & Aço_ACV & $\mathrm{kg}$ & $+7 \%$ \\
\hline & Poliruetano & Poliruetano & $\mathrm{kg}$ & $+1 \%$ \\
\hline & Vidro & Vidro_ACV & $\mathrm{kg}$ & $-2 \%$ \\
\hline \multirow{2}{*}{ Cobertura } & Telhado verde & Telhado_verde & $m^{2}$ & $-1 \%$ \\
\hline & Sistema de impermeabilização & Telhado_membrana & $m^{2}$ & $0 \%$ \\
\hline \multirow{4}{*}{$\begin{array}{l}\text { Divisórias } \\
\text { internas }\end{array}$} & Placa de gesso & Gesso_ACV & $\mathrm{kg}$ & $+8 \%$ \\
\hline & Madeira compensada & Madeira compensada_ACV & $m^{3}$ & $+3 \%$ \\
\hline & Madeira & Madeira_ACV & $m^{3}$ & $+9 \%$ \\
\hline & Alumínio & Alumínio & $m^{2}$ & $-7 \%$ \\
\hline Forro & Forro de gesso & Gesso_forro & $\mathrm{kg}$ & $+9 \%$ \\
\hline Piso externo & Concreto permeável & Concreto externo & $m^{3}$ & $0 \%$ \\
\hline \multirow{5}{*}{ Hidrossanitário } & Tubos de cobre (água) & Cobre (água) & $\mathrm{kg}$ & $-3 \%$ \\
\hline & Tubos de aço galvanizado (água) & Aço galvanizado (água) & $\mathrm{kg}$ & $-2 \%$ \\
\hline & Tubo de PVC (água) & PVC (água) & $\mathrm{kg}$ & $+1 \%$ \\
\hline & Tubo de PVC (esgoto) & PVC (esgoto) & $\mathrm{kg}$ & $+29 \%$ \\
\hline & Tubo de PPR (água + esgoto) & PPR & $\mathrm{kg}$ & $+80 \%$ \\
\hline \multirow{2}{*}{ AVAC } & Duto de aço galvanizado & Aço galvanizado & $\mathrm{kg}$ & $+7 \%$ \\
\hline & Lã de vidro & Lã de vidro & $\mathrm{kg}$ & $+3 \%$ \\
\hline \multirow{3}{*}{ Elétrico } & Eletroduto de aço galvanizado & Aço galvanizado (eletroduto) & $\mathrm{kg}$ & $-4 \%$ \\
\hline & $\begin{array}{c}\text { Fio elétrico - isolamento PVC + } \\
\text { camada de proteção }\end{array}$ & Isolante (fio elétrico) & $\mathrm{kg}$ & $+5 \%$ \\
\hline & Fio elétrico de cobre & Cobre (fio elétrico) & $\mathrm{kg}$ & $+2 \%$ \\
\hline & \multicolumn{3}{|c|}{ Variação média em relação ao quantitativo de referência } & $+6 \%$ \\
\hline
\end{tabular}

"Variação positiva indica que o quantitativo obtido por extração automática foi superior ao manual (referência). Apenas os materiais que foram também quantificados pelo método tradicional estão listados.

Fonte: Elaborado pelas autoras

As variações em cada subsistema foram, de modo geral, discretas (até 12\%). Apenas as tubulações de esgoto em PPR (80\%) e PVC (29\%) mostraram valores extraídos automaticamente muito superiores à quantificação de referência. Isso indica, por um lado, falha informacional na documentação desse projeto específico dadas as baixas variações registradas para tubulações de água em cobre, PVC e aço galvanizado. Por outro, ressalta a superioridade 
da modelagem BIM nos casos de projetos incompletos, que podem prejudicar a confiabilidade de resultados justamente em elementos de grande impacto ambiental, como, no caso, componentes plásticos.

A variação discreta observada confirma a validade da extração automática para realização de ACV e sugere a modelagem em LOD 300 como alinhada ao nível de acurácia praticado atualmente, em geral, a partir de projetos básicos detalhados.

O procedimento de inserção de parâmetros para cálculo ambiental dentro do ambiente BIM elimina etapas e interfaces presentes na avaliação convencional, como representado em notação BPMN na Figura 4, que exemplifica os impactos de energia e emissões incorporadas. O modelo é gerado de acordo com as metas e os objetivos da ACV, previamente estabelecidos desde a concepção do projeto. $\mathrm{O}$ trabalho integrado do time de projeto soma-se à extração automática de dados para tornar a ACV mais precisa e consistente. As principais modificações ocorrem no processo de projeto em si e no procedimento de coleta de dados para ACV, principalmente no reordenamento das atividades desenvolvidas e na adição e alteração das informações trocadas. Já os agentes envolvidos não sofrem alterações.

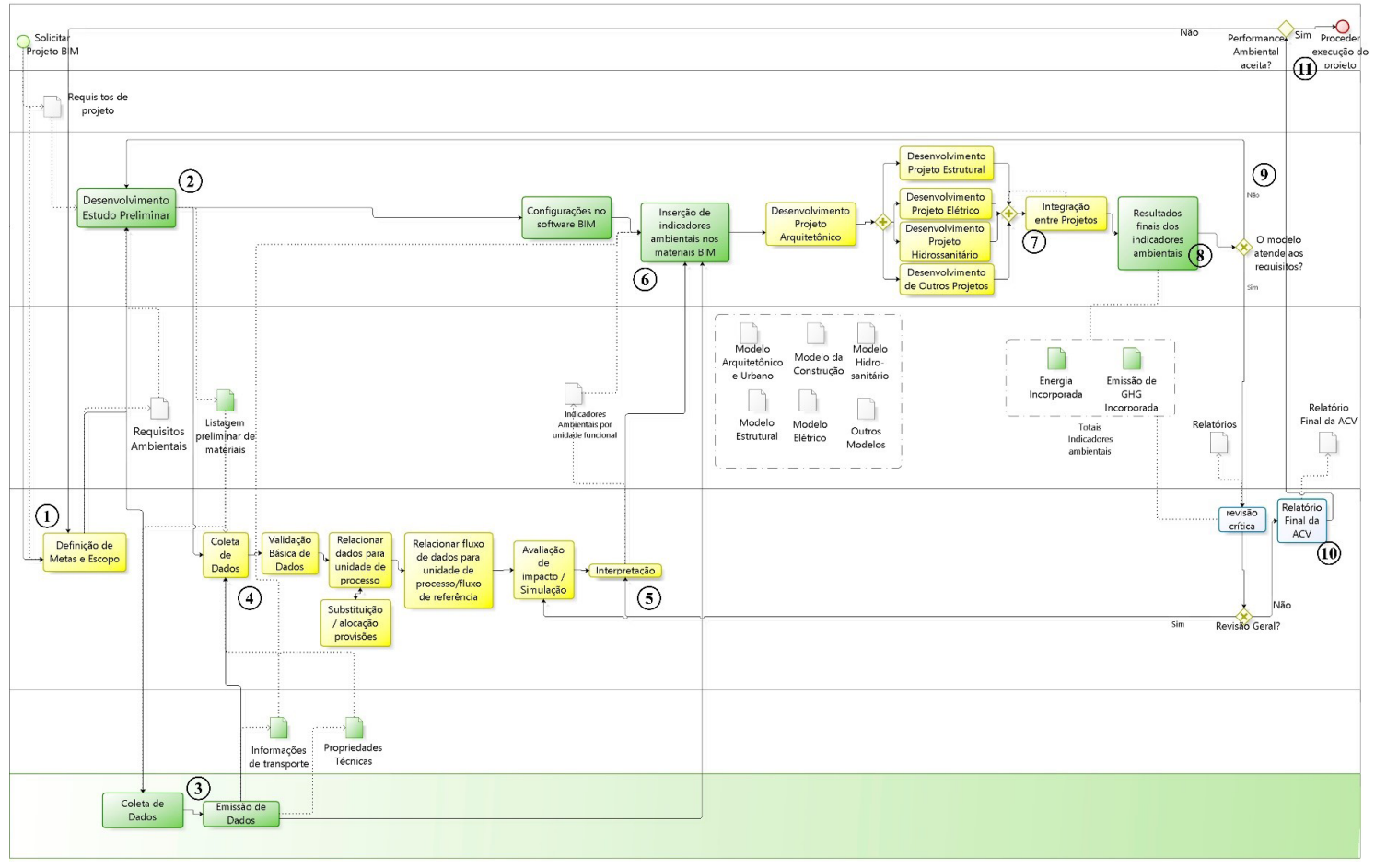

Figura 4: Processo de projeto auxiliado por BIM e orientado à ACV de edificações (BIM4LCA): mapa geral otimizado

Fonte: Barros; Silva, 2016
Na proposição completa feita por Barros e Silva (2016), após a solicitação do projeto pelo cliente, o especialista ACV define a meta e o escopo (1) para desenvolvimento do estudo preliminar (2). Os dados referentes aos materiais de construção que possivelmente serão utilizados são acrescidos das informações relativas ao transporte desde um potencial fornecedor (3), e o especialista ACV realiza a avaliação (4) e calcula coeficientes de impacto por unidade funcional (5). Esses coeficientes são repassados à equipe de projeto, juntamente com distância e tipo de transporte desde fornecedores, para inserção nos elementos BIM (6). Os projetos são desenvolvidos (7), e os indicadores ambientais, (re)calculados dentro do próprio software BIM (8). Caso o projeto não atenda aos requisitos ambientais, o modelo retorna à etapa anterior para revisão (9), até que a ACV possa ser finalizada (10). Caso o desempenho ambiental seja aceito pelo empreendedor, procede-se à execução do projeto (11). Caso contrário, retorna-se à etapa inicial. 
Por se tratar da elaboração de um modelo BIM a partir de documentação de um projeto - em vez do produto do processo real de desenvolvimento projetual - nosso estudo de caso não pode implementar completamente esta proposição e aproveitar o maior benefício conceitual do emprego de modelagem da informação para otimização de desempenho ambiental da edificação: o fornecimento de dados, já nas fases iniciais de projeto, são suficientes para testar e orientar a tomada de decisão por materiais e componentes mais sustentáveis. Ainda assim, foi possível demonstrar que modelos podem ser preparados para facilitar $\mathrm{ACV}$ de forma operacionalmente simples, ainda que trabalhosa, pela inserção de indicadores e execução de cálculos ambientais dentro do software BIM. A automatização futura desse procedimento através de uma tecnologia ETL pode melhorar sobremaneira o processo, ainda que reste a maior dificuldade que hoje pressiona a ACV na maioria dos países, de alimentar os bancos de dados requeridos com inventários de ciclo de vida contextualizados.

\section{CONCLUSÕES}

A modelagem BIM pode facilitar a avaliação do ciclo de vida de edificações e auxiliar na escolha de materiais. Este artigo explorou contribuições da modelagem BIM para facilitar o processo de ACV de edificações completas. Verificamos que LOD 300 é compatível com o nível de acurácia praticado atualmente em ACV. É possível que LOD inferiores possam ser trabalhados em etapas de concepção e estudo preliminar, mas essa avaliação não foi parte do escopo deste artigo. Já a necessidade de empregar LOD superiores é menos provável, dadas as incertezas e a limitação da disponibilidade de dados de inventário intrínsecos ao estado atual da ACV de edificações, mas podem ser úteis para realizar, por exemplo, uma auditoria ambiental de um empreendimento específico, guiada pela inserção precisa de informações a ele referentes.

O método comumente utilizado de extração automática dos quantitativos de materiais a partir do modelo BIM foi validado para fins da ACV e traz ganhos de tempo, esforço de coleta de dados, e redução de risco de erros durante 0 processo manual, sem obstruir o processo de projeto. Mais ainda, no modo BIM4LCA, o modelo pode ser preparado para facilitar ACV e já realizar os cálculos dentro do modelo de modo a se extrair listagens de impactos em vez de simples quantitativos, aumentando significativamente a contribuição de BIM. Essa preparação é operacionalmente simples, mas de grande efetividade. No desenvolvimento de novos projetos, esse procedimento pode fornecer feedback rapidamente - ainda que unidirecional - e fundamentar uma tomada de decisão mais robusta para elevar desempenho ambiental de edificações, enquanto interoperabilidade completa ou mesmo o emprego de tecnologias ETL não se tornarem prática comum em Aeco.

\section{AGRADECIMENTOS}

As autoras agradecem a Capes e CNPq pelo apoio financeiro concedido.

\section{REFERÊNCIAS}

ALWAN, Z.; JONES, P. The importance of embodied energy in carbon footprint assessment. Structural Survey, London, v. 32, n. 1, p. 49-60, 2014

AMERICAN INSTITUTE OF ARCHITECTS. Guide, instructions and commentary to the 2013 AIA digital practice documents. Washington, DC, 2013.
ASSOCIAÇÃO BRASILEIRA DE NORMAS TÉCNICAS. NBR ISO 14040: Gestão ambiental: avaliação do ciclo de vida: princípios e estrutura. Rio de Janeiro, 2009a.

NBR ISO 14044: Gestão ambiental: avaliação do ciclo de vida: requisitos e orientações. Rio de Janeiro, 2009b. 
AZHAR, S. BIM for sustainable design: results of an industry survey. Journal of Building Information Modeling, Winnipeg, v. 4, n. 1, p. 27-28, 2010.

AZHAR, S. et al. Building information modeling for sustainable design and LEED ${ }^{\circledR}$ rating analysis. Automation in Construction, Amsterdam, v. 20, n. 2, p. 217-224, mar. 2011.

BARROS, N. N. Impactos da adoção de BIM na avaliação de energia e emissões de GHG incorporadas no ciclo de vida de edificações. 2016. Dissertação (Mestrado em Arquitetura, Tecnologia e Cidade) Faculdade de Engenharia Civil, Arquitetura e Urbanismo, Universidade Estadual de Campinas, Campinas, 2016

BARROS, N. N.; SILVA, V. G. BIM na avaliação do ciclo de vida de edificações: revisão da literatura e estudo comparativo. PARC Pesquisa em Arquitetura e Construção, Campinas, v. 7, n. 2, p. 89-101, jun. 2016. doi: 10.20396/parc.v7i2.8645401. Disponível em: <https://goo.gl/ydg19o>. Acesso em: 30 jun. 2016.

BAUMANN, H.; TILLMAN, A. M. The Hitch Hiker's guide to LCA. Lund: Studentlitteratur AB, 2004.

BECERIK-GERBER, B.; KENSEK, K. Building information modeling in architecture, engineering, and construction: emerging research directions and trends. Journal of Professional Issues in Engineering Education and Practice, Reston, v. 136, n. 3, p. 139-147, 2010.

BEDRICK, J. A level of development specification for BIM processes. AECbytes, 16 maio 2013. Disponível em: <https://goo. gl/x5sFS7>. Acesso em: 22 jul. 2015.

BRITISH STANDARDS INSTITUTION. BS EN 15978:2011. Sustainability of construction works: assessment of environmental performance of buildings: calculation method. London, 2011.

BYNUM, P.; ISSA, R.; OLBINA, S. Building information modeling in support of sustainable design and construction. Journal of Construction Engineering and Management, Reston, v. 139, n. 1, p. 24-34, 2013

DIAZ, J.; ANTON, L. Á. Sustainable construction approach through integration of LCA and BIM tools. In: COMPUTING IN CIVIL AND BUILDING ENGINEERING - PROCEEDINGS OF THE 2014 INTERNATIONAL CONFERENCE ON COMPUTING IN CIVIL AND BUILDING ENGINEERING, 23-25 jun. 2014, Orlando. Conference Paper... Orlando: 2014. Disponível em: <https://goo.gl/yPsEyK>. Acesso em: 23 mar. 2018

EASTMAN, C. et al. BIM handbook: a guide to building information modeling for owners, managers, designers, engineers and contractors. Hoboken: John Wiley \& Sons, 2008

IDDON, C. R.; FIRTH, S. K. Embodied and operational energy for new-build housing: a case study of construction methods in the UK. Energy and Buildings, Amsterdam, v. 67, p. 479-488, dez. 2013

JRADE, A.; JALAEI, F. Integrating building information modelling with sustainability to design building projects at the conceptual stage. Building Simulation, New York, v. 6 , n. 4, p. 429-444, dez. 2013.

KRYGIEL, E.; NIES, B. Green BIM: successful sustainable design with building information modeling. Hoboken: John Wiley \& Sons, 2008.

LEITE, F. et al. Analysis of modeling effort and impact of different levels of detail in building information models. Automation in Construction, Amsterdam, v. 20, n. 5 , p. 601-609, ago. 2011.

MCGRAW-HILL CONSTRUCTION. Building information modeling: transforming design and construction to achieve greater industry productivity. New York, 2008.

MEADATI, P.; IRIZARRY, J.; AKHNOUKH, A. Building information modeling implementation-current and desired status. In: Computing in Civil Engineering. Reston: American Society of Civil Engineers, 2011. p. $512-519$.

OBJECT MANAGEMENT GROUP. Business Process Model and Notation (BPMN): version 2.0. Needham, 2011.

PENG, C. Calculation of a building's life cycle carbon emissions based on Ecotect and building information modeling. Journal of Cleaner Production, Amsterdam, v. 112, p. 453-465, 2014.

RIST, T. A path to BIM-based LCA for wholebuildings. Master - Spring: Norweigan University of Science and Technology, 2011.

SAADE, M. R. M. Influência da alocação de impactos na indústria siderúrgica sobre a avaliação de ciclo de vida de cimentos. Campinas: Unicamp, 2013.

SCHADE, J. et al. A comparative study of the design and construction process of energy efficient buildings in Germany and Sweden. Energy Policy, Amsterdam, v. 58, p. 28-37, 2013. doi: 10.1016/j.enpol.2013.02.014

SHADRAM, F. et al. An integrated BIM-based framework for the energy assessment of building upstream flow. In: INTERNATIONAL CONFERENCE ON CONSTRUCTION AND REAL ESTATE MANAGEMENT, 11-12 ago. 2015, Lulea. Anais... Reston: American Society of Civil Engineers, 2015. doi: 10.1061/9780784479377.013 
SHADRAM, F. et al. An integrated BIM-based framework for minimizing embodied energy during building design. Energy and Buildings, Amsterdam, v. 128, p. 592-604, 2016. doi: 10.1016/j.enbuild.2016.07.007

SHADRAM, F.; MUKKAVAARA, J. An integrated BIM-based framework for the optimization of the trade-off between embodied and operational energy. Energy and Buildings, Amsterdam, v. 158, p. 1189-1205, 2018. doi: 10.1016/j.enbuild.2017.11.017.

SILVA, V. G. et al. Life beyond operational stage: exploring lifecycle zero energy definitions. In: IISBE NET ZERO BUILT ENVIRONMENT 2014 SYMPOSIUM, 6-7 mar. 2014, Gainesville. Proceedings... Gainesville: University of Florida: Powell Center for Construction \& Environment, 2014.
SOUST-VERDAGUER, B.: LLATAS, C.; GARCÍAMARTÍNEZ, A. Critical review of bim-based LCA method to buildings. Energy and Buildings, Amsterdam, v. 136, p. 110-120, 2017. doi: /10.1016/j.enbuild.2016.12.009

VANLANDE, R.; NICOLLE, C.; CRUZ, C. IFC and building lifecycle management. Automation in Construction, Amsterdam, v. 18, n. 1, p. 70 78, 2008. doi: 10.1016/j.autcon.2008.05.001

YUNG, P.; WANG, X. A 6D CAD model for the automatic assessment of building sustainability. International Journal of Advanced Robotic Systems, Thousand Oaks, v. 11, n. 1, 2014.

WONG, K.; FAN, Q. Building information modelling (BIM) for sustainable building design. Facilities, Bingley, v. 31, p. 138-157, 2013. doi: $10.1108 / 02632771311299412$
Vanessa Gomes

vangomes@gmail.com

Natalia Nakamura Barros

natalianakamura.ara@gmail.com 
